\title{
Spirituality/Religiosity as a Resource for Coping in Soldiers: A Summary Report
}

\author{
Arndt Büssing, MD
}

\begin{abstract}
Background: Individuals often reflect on their future perspectives and vulnerable concepts of life, and ask for meaning and purpose in life, especially when confronted with stressful experiences and subsequent physical impairments and/or mental afflictions. Spirituality/religiosity $(\mathrm{SpR})$ is a relevant resource used by individuals to cope with such events.

Objective: This summary report focuses on $\mathrm{SpR}$ as a resource that Soldiers can use to deal with stressful situations and combat-associated health afflictions.

Method: This summary presents information on specific studies indicating that spirituality can be a relevant resource for traumatized Soldiers. After a combat experience, for a Soldier, an assessment of his or her unmet psychosocial and spiritual needs might be particularly appropriate because this (low threshold) assessment avoids stigmatization of the Soldier as a "weak" person who would require psychologic/psychotherapeutic help. In the Military context, it is essential to recognize that SpR may be a beneficial resource to aid coping and adaptation.

Conclusions: Both chronic illness and post-traumatic stress have significant impacts on life concerns and affect physical, functional, emotional, social, and spiritual well-being. Research has shown that SpR may have multiple beneficial effects on health and performance. Moreover, the ability to reflect priorities and change attitudes and behavior in response to illnesses/traumas could be an important factor for long-term adjustment, even if symptoms may persist.
\end{abstract}

Key Words: Spirituality, Religiosity, Coping, Resources, Soldiers, Health Afflictions

\section{INTRODUCTION}

W HEN CONFRONTED with stressful experiences and subsequent physical impairments and/or mental afflictions, some individuals reflect on their future perspectives and vulnerable concepts of life; ask for meaning and purpose in life; and consider which beneficial sources to rely on (e.g., religion). Affected individuals may ask for helpful spiritual resources that can provide secure havens for these individuals' struggles with illness or impairments. In fact, for many people confronted with traumas or chronic diseases, spirituality/religiosity ( $\mathrm{SpR})$ is a relevant resource to assist with coping - even in secular societies. A special issue of the journal Military Medicine addressed spirituality as a relevant aspect of a multidimensional Total Force Fitness model of the U.S. Army. This model includes nutritional, psychologic, behavioral, social, physical, environmental, medical, and spiritual components. ${ }^{1}$ In that special issue, Hufford et al. clearly stated that:

Professorship Quality of Life, Spirituality and Coping, Institute of Integrative Medicine, Witten/Herdecke University, Herdecke, Germany.

This article was prepared as part of the work product of NATO Science and Technology Organization, Task Force, Human Factors and Medicine (STO-TR-HFM) 195, Integrative Medicine Interventions for Military Personnel. 
for many on the front lines, spirituality and religion are the only "safe haven" amidst intense operational or combat experiences that can test one's faith. The danger of spiritual and moral trauma is real, and it can initiate a downward spiral of physical, psychological, and behavioral problems in the Service Member. ${ }^{2}$

In this chapter, essential findings from scientific studies on the relevance of $\mathrm{SpR}$ (as an attitude) for coping with chronic illness and trauma are described. Then, the putative impact of SpR as a resource for coping is discussed in the Military context. Spirituality-based interventions such as mindfulness meditation are outlined in a separate article in this issue, "Mindfulness Based Practices as a Resource for Health and Well-Being," pages 349-359, and are not addressed here.

\section{DEFINITIONS OF SpR}

There are various definitions of what $\mathrm{SpR}$ is or might be. Depending on the cultural context, religious denomination, worldview, or profession, these definitions may vary significantly.

First, spirituality and religiosity are often used as interchangeable terms; yet, from a conceptual point of view, they are not identical. Spirituality is a complex and multidimensional construct and can be defined as an open and individual experiential approach in the search for meaning and purpose in life ("content"), while religion is an institutional and culturally determined approach that organizes the collective experiences of people (faith) into a closed system of beliefs and practices ("form"). ${ }^{3}$ Spirituality can be found through religious engagement, through an individual experience of the Divine, and/or through a connection to other people, the environment and the Sacred. Moreover, one should differentiate specific beliefs (cognition/emotion), well-being in the context of spirituality and/ or religiosity, and specific SpR practices (actions), either within specific institutional context or highly individual approaches.

One of the more general and broad definitions of spirituality, for example is as follows:

Spirituality refers to an attitude of search for meaning in life. The searching individual is aware of its divine origin (either transcendent or immanent, i.e., God, Allah, JHWH, Tao, Brahman, Prajna, Unity etc.), and feels a connection with others, nature and the Divine. Because of this awareness one strives towards the realization of the respective teachings, experiences or insights, which have a direct impact on conduct of life and ethical commitments. $^{3}$
This can be reduced to six subsequent practical principles that can be a framework also for the Military context because it implies also the topics of morality/ethics and the demand to help: (1) Life has meaning. (2) We share the same origin. (3) We are connected. (4) We have a responsibility. (5) We have an ethical commitment. (6) We protect and shelter.

Today, spirituality is globally attributed to humans' search for meaning in life-which can but must not involve rituals and practices concerning a higher power or God. ${ }^{4}$ Other people would define spirituality as a search for "transcendental truth" that may include a sense of connectedness with other people, nature, and/or the Divine, and thus "spirituality can call us beyond self to concern and compassion for others." 5 In addition, Pargament argued that spirituality is the search for significance in ways related to the Sacred. ${ }^{6}$ This specific Sacred can be highly individual, and must not necessarily be identical to those of religious denominations.

In a more secular context, spirituality can be viewed as a general concept that may include specific forms of religiosity but shares defined aspects with secular forms of spirituality as well. While this differentiation is important in countries with secular and liberal backgrounds (i.e., Europe), this distinction is meaningless in countries with conservative theistic beliefs.

A summary of this follows:

Spirituality is not only the "experiential core" (content) of ritualized religiosity (form), but a complex construct which shares relevant topics with secular aspects of spirituality. The underlying motifs found in the various definitions are the (cognitive) "search for meaning," the (emotional) experience of connectedness (with God, others, and nature), and the respective realization in daily life in accordance with related ethical conduct. One may presume that spirituality could be assumed as persons' commitment to a higher principle/source that is embodied their daily [lives]. ${ }^{7}$

This is also in accordance with the definition for spirituality, which the Army Chief of Chaplains utilizes, "a process transcending self and society that empowers the human spirit with purpose, identity, and meaning." ${ }^{\prime} *$

\section{ASSOCIATIONS BETWEEN SpR AND HEALTH-RELATED VARIABLES}

There are an increasing number of studies showing that specific aspects of SpR can be positively associated with health-related variables. ${ }^{9}$ Although these results are not always consistent and are often dependent on circumscribed populations, distinct cultures, and specific measures, many studies have found positive associations among defined

*The information for ref. 8 was provided by an e-mail from a staff member at the Center for Spiritual Leadership (CSL), at the U.S. Army Chaplain Center and School, in Fort Jackson, SC on May 14, 2010. 
facets of SpR and psychologic well-being, ${ }^{10}$ quality of life (QoL), ${ }^{11}$ and coping. ${ }^{12}$ A systematic review on the "potential beneficial or harmful effects of religious/spiritual coping" indicated that this specific form of coping may be beneficial to "maintaining self-esteem, providing a sense of meaning and purpose, giving emotional comfort and providing a sense of hope."13

Moreover, SpR engagement might also have positive effects on health. A recent meta-analysis (which enrolled 69 studies with healthy populations and 22 studies with patients) showed that SpR was "associated with reduced mortality in healthy population studies (combined hazard ratio $=0.82$, 95\% CI (confidence interval) [0.76-0.87], $p<0.001$ ), but not in diseased population studies (combined hazard ratio $=0.98$, 95\% CI [0.94-1.01], $p=0.19) . "{ }^{14}$ Interestingly, this metaanalysis indicated that organizational activities (i.e., church attendance, engagement in community, etc.) were positively associated with reduced mortality, not with intrinsic aspects (convictions, experiences, etc.). This may indicate that SpR engagement can have preventive effects.

Further evidence comes from a systematic review that investigated the cancer risk among members of Christian communities. ${ }^{15}$ The 17 included epidemiologic studies indicated a reduced cancer risk only for lifestyle-associated cancers (i.e., those associated with tobacco smoking, alcohol consumption, diet, physical activity, and reproductive factors). Interestingly, the most important factor for this effect was the healthy lifestyle inherent in religious practices in these communities. ${ }^{15}$ This means specific SpR attitudes and convictions and subsequent behavior and activities can be associated with healthy lifestyles, albeit this will not necessarily guarantee health, prevention of illness, or rapid recovery. Rather, it is true that $\mathrm{SpR}$ is one factor among several biopsychosocial variables that may contribute to improved health and QoL.

Although patients, physicians, and chaplains may have high expectations, SpR cannot be used like an automatic "wishing well" and cannot be prescribed. SpR requires a stable inner conviction (belief/faith) that has been proven by positive experiences during life. Then, in case of need, one may rely on this resource to cope with illness and suffering.

Indeed, trauma survivors relying on SpR beliefs for coping may show a greater ability for post-traumatic growth (i.e., greater appreciation of life and changed priorities, warmer, more-intimate relations with other people, a greater sense of personal strength, recognition of new possibilities, and spiritual development). ${ }^{16}$ Moreover, even after spinalcord injury several patients have had positive experiences. Contributing factors to facilitating positive views were, personality (confidence, assertiveness, independent person), support systems (family, friends, and/or health care professionals), spiritual connectivity (hope and strength to continue with life), and acceptance of one's disability (many of these patients felt that acceptance was ongoing, requiring time and reflection). ${ }^{17}$
Thus, patients may use their SpR foremost as a "strategy" to cope with illness and suffering, ${ }^{6}$ even in a secular society. ${ }^{18-21}$ Whether this SpR can be objectified or not, it is important that patients with chronic diseases regard their $\mathrm{SpR}$ as beneficial for: managing their lives more consciously; promoting inner strength; providing feelings of inner peace; attaining deeper connections with other people and the surrounding world; coping better with illness; and even restoring one to mental and physical health. ${ }^{22}$

However, one has to face the fact that a growing number of individuals in Western societies reject institutional religiosity but may have an interest in more secular and individual approaches. Here, specific aspects of spirituality such as Conscious interactions (with other people, self, and environment) and Compassion/Generosity are of higher relevance than a conventional religious orientation. ${ }^{23}$ These specific aspects can be regarded as shared values among different religious and cultural approaches, and are relevant even for nonreligious skeptics.

\section{SPIRITUAL NEEDS}

Although some individuals may have lost faith in institutional religiosity, they may nevertheless have specific spiritual needs-which are, in most cases, neither recognized nor addressed by health care professionals. A recent study among 230 patients with advanced cancer has shown that a majority of these patients $(72 \%)$ reported that their spiritual needs were supported minimally or not at all by the medical system, while $47 \%$ of these patients reported that they felt supported minimally or not at all even by a religious community. ${ }^{24}$ This means a large proportion of patients are left alone with unmet spiritual needs, because no one feels responsible or competent to address these needs.

In addition, a survey among more secular German patients with chronic pain conditions revealed that $23 \%$ of these patients talked with chaplains/priests about SpR needs, $20 \%$ had no partners to talk with about these needs, and $37 \%$ stated that it was important to talk with their medical doctors about these needs. ${ }^{19}$ Yet, most physicians lack the necessary time and skills to address their patients' spiritual needs. However, this support is essential for such patients, because supporting these needs by medical teams and via pastoral-care visits was significantly associated with patients' QoL. ${ }^{25}$

If it is true that some patients do have psychosocial and spiritual needs that are important to them, then these needs have to be identified. Only when these needs are identified, can health care professionals, Military supervisors, and patients' relatives have the chance to react to these needs and support these patients in their struggles with health affections or even chronic and fatal diseases.

For research and clinical practice, a conceptual framework was suggested that categorizes four (interconnected) 
core dimensions of psychosocial and spiritual needs: (1) Connection (i.e., love, belonging, alienation, partner communication, etc.); (2) Peace (i.e., inner peace, hope, balance, forgiveness, distress, etc.); (3) Meaning/Purpose (i.e., meaning in life, self-actualization, role function, etc.); and (4) Transcendence (i.e., spiritual resources, relationship with God/Sacred, praying, etc.), which can be attributed to the underlying categories of social, emotional, existential, and religious concerns. ${ }^{26}$

In U.S. patients with cancer, spiritual and existential needs were specified by these patients' intentions to obtain help for overcoming fears, and to find hope, meaning in life, spiritual resources, or other people to talk with about finding peace of mind. ${ }^{27}$

In German patients with chronic pain diseases and cancer $(N=392)$, Religious needs and Existential needs were scored relatively low, while needs for Inner Peace and Giving/ Generativity were scored high. The needs for Giving/Generativity are particularly of interest, because they address the intention to give solace to other people, to transmit information about one's own life experiences to other people, and to be assured that life was meaningful and valuable. ${ }^{28}$ This ability to care for others, to guide the next generation, and to know that one's own actions and decisions were meaningful, is of outstanding importance also for traumatized Soldiers. Similar findings were observed in predominantly atheistic patients from Shanghai ${ }^{29}$ and Catholic patients from Poland. ${ }^{30}$ Interestingly, particularly this generativity aspect of spirituality connects to three functions of the U.S. Army chaplaincy's definition of spirituality (i.e., empowering people with purpose, identity, and meaning). ${ }^{8^{*}}$

Thus, individuals with chronic health afflictions predominantly report needs related to inner peace and generative relatedness on personal levels, whereas needs associated with transcendent relatedness were of minor relevance-at least in secular societies. Nevertheless, even nonreligious skeptics can express specific religious needs, and these should be recognized.

\section{SPIRITUALITY IN THE MILITARY CONTEXT}

There are only a few empirical studies addressing the relevance of SpR in a Military setting. A survey of World War II veterans, two recent studies, and data from a study among German Soldiers are highlighted below. ${ }^{8,31-33}$

A survey of World War II Veterans (of a random national sample of 7500 persons) revealed that church attendance occurred more often among Veterans who had faced heavy combat and claimed that their war experience was negative, while those who claimed their experience was positive attended church less often. ${ }^{31}$ Thus, negative combat experience was related to religious practice. One may suggest that this is a strategy for obtaining relief.
Referring to data in the "2008 Department of Defense Survey of Health Related Behaviors Among Active Duty Military Personnel" (including datasets of 28,546 participants), Hourani and coworkers reported that spirituality buffered against depression and post-traumatic stress disorder (PTSD) symptoms only among Military personnel with lowmoderate combat exposure, while a medium level of spirituality was protective against self-reported suicidal ideation/ attempts. Interestingly, the researchers stated that "high levels of spirituality may be associated with greater suicidal ideation or attempt in this nondeployed subgroup of Military personnel.," ${ }^{32}$ The implication of this surprising finding is currently unclear. However, spirituality was measured with two items only (importance of religious/spiritual beliefs and influence of religious/spiritual beliefs on decision making), which is pragmatic for such a large-scale study, albeit a highly reduced approach to address a multifaceted dimension such as spirituality. Moreover, the psychologic profile of these "high spirituality" persons was unclear. It might be, as stated by the researchers, that this "nondeployed group is coming into the service with higher levels of suicidal ideation." The findings of Hourani et al.'s study point clearly to the fact that "the buffering role of spirituality in mental health is limited" and that this effect "may be potentially overwhelmed by great stress, such as high levels of combat exposure." 32

A further study, "The Army's Excellence in Character, Ethics, and Leadership (EXCEL) survey," refers to data for 1366 soldiers with combat experience in Iraq (collected in the summer of 2009). ${ }^{8}$ For this study, a multidimensional approach to measure spirituality with 15 items and three underlying factors were used (i.e., (1) Connection to Others; (2) Religious Identification, and (3) Hopeful Outlook). While there were no significant correlations between these three measures and the number of deployments, higher spirituality scores correlated weakly with age and rank. ${ }^{8}$ More interestingly, spirituality correlated moderately with variables such as Moral Courage/Ownership, Moral Efficacy, Embracing Army Values, Intent to Report Unethical Conduct, and Soldier Identification. However, Religious Identification correlated less strongly than Hopeful Outlook or Connection to Others. A further important finding of this study was that Soldiers' emotional resilience was moderately associated with their spirituality, particularly with Hopeful Outlook, while physical and psychologic fatigue was only marginally and inversely associated with spirituality. ${ }^{8}$

A recent study enrolling German soldiers $(N=1092)$ addressed their psychosocial spiritual needs and mental health affections (Büssing \& Recchia, 2015). ${ }^{33}$ The data are in line with findings among German patients with chronic diseases (i.e., Religious Needs and Existential Needs scored low in the enrolled Soldiers, while Inner Peace Needs and Giving/Generativity Needs were of higher relevance). In particular, Existential Needs and Inner Peace Needs correlated moderately with Soldiers' perceived stress (PSS scale) and PTSD symptoms (PCL-M scale), and inversely with life 
satisfaction. Giving/Generativity needs were moderately associated with perceived stress and PTSD symptoms. In contrast, Religious Needs were only marginally associated with stress and PTSD symptoms, but not with life satisfaction. ${ }^{33}$ This means that, although most German Soldiers reported no specific Religious or Existential Needs, half of them had Inner Peace Needs and Giving/Generativity Needs. Because these needs were significantly correlated with health afflictions and reduced life satisfaction, these needs require further attention, as they may indicate an opportunity for Soldiers to obtain support without facing a stigmatizing psychotherapeutic treatment.

These recent studies show that the relational and hopeassociated aspects of spirituality were particularly associated with Soldiers' ethical attitudes and personal resilience, while the self-ascribed importance of religious/spiritual beliefs and their influence on decision making were not necessarily a buffer against depression or PTSD symptoms. In fact, $\mathrm{SpR}$ as an attitude can be a guideline for ethical decisions and moral behavior, can be helpful for coping with suffering, and can be a resource for hope. Indeed, "religious moral beliefs" of 130 male war veterans from Bosnia and Herzegovina were helpful for protecting their mental health after surviving multiple war traumas. ${ }^{34}$ Yet, SpR is by no means a "tool" for creating health or a buffer against any harm. Rather, it is true that specific spiritual needs may indicate psychoemotional problems, which could be addressed very early to prevent health afflictions and Service failure.

\section{HELP-SEEKING FROM SPIRITUAL COUNSELORS AND CLERGY}

In the U.S. and Canadian armies, soldiers' help-seeking behavior is encouraged, because (silent) mental health afflictions in response to stressful missions ("suffering while functioning") are no longer regarded as stigmas. Nevertheless, there are still several barriers, both structural and individual (i.e., the attitude of "self-management," and thus masked impairment) which makes it difficult to identify actual needs.

Nevertheless, Soldiers and veterans are seeking help for depression and PTSD from spiritual counselors and clergy. Bonner et al. screened Veterans with probable major depression and PTSD $(N=761)$ and found that $47 \%$ of them “endorsed being 'very' or 'somewhat likely' to seek help for emotional problems from spiritual counselors," while $12 \%$ reported "actual spiritual counselor/clergy consultation." 35 Thus, the researchers recommended integrating spiritual counselors/clergy into care teams. Moreover, some Veterans at risk for suicide were actively seeking pastoral care, "demonstrating a demand for such services.",35

\section{DISCUSSION}

Both chronic illness and post-traumatic stress have a significant impact on life concerns and affect physical, functional, emotional, social, and spiritual well-being. Research has suggested that SpR may have multiple beneficial effects on health and performance. Moreover, the ability to reflect priorities, and change attitudes and behavior, in response to illness/trauma could be an important factor for long-term adjustment, even if symptoms may persist. There is no doubt that specific beliefs (which may provide support in times of need), values (which also have an influence on health and risky behaviors), and practices/engagement (which may provide psychoemotional comfort) may contribute to mental stability. Yet, engagement in specific SpR issues-or even strong religious beliefs-is by no means a "preventive protection" against combat damage or health afflictions.

It might be a misinterpretation of research data when one aims to utilize spirituality as a preventive tool. So far, it is unclear whether or not specific training programs aiming to improve Soldiers' "spiritual fitness," - such as the Comprehensive Soldier Fitness program of the U.S. Army, which involves the core dimensions Physical, Emotional, Social, Family, and Spiritual (including strengthening beliefs and values) - to strengthen Soldiers' general resilience is effective with respect to the intended aims (i.e., reducing PTSD or suicide risk). ${ }^{36}$ Although there is no doubt that such programs can be of value for those who share the same beliefs; Soldiers who reject these specific forms of Christianity may have problems with it.

One has to recognize that human beings have specific attitudes, convictions, and beliefs that may differ with respect to respective cultural and religious contexts. Simply assessing whether a Soldier has a specific belief or not, can be a first step; yet, it is not enough to assess a complex and multifaceted construct such as $\mathrm{SpR}$.

Table 1. Illustration of the Complex Interplay of Health, Illness, and Recovery Determinants

\begin{tabular}{|c|c|c|c|c|c|}
\hline Challenges & Predispositions & Resources & & Coping behavior & Health status \\
\hline$\rightarrow$ & & $\rightarrow$ & Spiritual needs & $\rightarrow$ & \\
\hline $\begin{array}{l}\text { Critical life events } \\
\text { Combat traumas }\end{array}$ & $\begin{array}{l}\text { Resilience } \\
\text { Ethics/values } \\
\text { Socialization } \\
\text { Education }\end{array}$ & $\begin{array}{l}\text { Social support } \\
\text { Self-efficacy } \\
\text { Mindfulness } \\
\text { Spirituality }\end{array}$ & & $\begin{array}{l}\text { Reactive coping strategies } \\
\text { Positive life construction }\end{array}$ & $\begin{array}{l}\uparrow \text { Quality of life } \\
\downarrow \text { Health afflictions/distress }\end{array}$ \\
\hline
\end{tabular}




\section{CONCLUSIONS}

In the Military context, it is essential to recognize that $\mathrm{SpR}$ is a beneficial resource for aiding coping and adaptation. Although not all Soldiers may share the same underlying beliefs, it is nevertheless of importance to acknowledge and support SpR attitudes and convictions of soldiers who rely on this resource.

\section{RECOMMENDATIONS}

Studies indicate that spirituality can be a resource of relevance for traumatized Soldiers. In particular, for Soldiers after combat experience, an assessment of their unmet psychosocial and spiritual needs might be appropriate because this (low threshold) assessment avoids stigmatization of any Soldier as a "weak" person who would need psychologic/psychotherapeutic help. An appropriate instrument to assess these needs might be the Spiritual Needs Questionnaire (SpNQ), $\dagger$ which is currently being used in a study among Soldiers, and which has already been validated for use with religious and nonreligious patients with chronic diseases. ${ }^{28-30,37}$ The individual responses to this instrument can be used to start a conversation or counseling regarding what these needs may mean to Soldiers and how they can be supported.

Medical doctors, psychologists and Military supervisors are not necessarily supposed to share the spiritual attitudes and convictions of their patients and subordinates, but these professionals should appreciate these attitudes and convictions and react adequately when specific needs are expressed. An appropriate support of specific spiritual needs may help improve relevant aspects of patients' QoL. Research shows clearly that health care professionals can play an important role in enhancing patients' psychospiritual well-being (i.e., self-awareness, coping and adjusting effectively to stress, relationships, sense of faith, sense of empowerment and confidence, and living with meaning and hope). ${ }^{38}$

To address Soldiers' spiritual needs, multiprofessional teams (i.e., psychologists, chaplains, nurses, and medical doctors) should care for the multifaceted needs of their patients/clients. However, Military supervisors and team comrades should be included in this process of care because they share the same responsibility to care for each other.

With respect to the vulnerability-stress model, one can integrate (and detect) various aspects of $\mathrm{SpR}$ and other beneficial resources along a path between stressors and health, which may be a conceptual framework for spiritual care approaches. Reactions to challenges (i.e., combat traumas) depend on the interaction of several predisposing factors involved (i.e., socialization, dispositions) and availability of social and individual resources (i.e., supportive community, family, team, specific beliefs/convictions), which may generate a basis for a sense of coherence to determine the handling of such challenges. Specific active coping strategies are the individual processes to handle the burden of the challenges. The complex interplay between these factors determines status of health, illness and recovery (Table 1).

With respect to palliative care, the Pasadena Consensus Conference held in 2009 advised clearly that "spiritual care should be integral to any compassionate and patient-centered health care system model of care," and recommended spiritual assessment of both patients and families. ${ }^{39}$ These recommendations can be transferred easily to spiritual care of persons who are not necessarily in palliative situations but who may require similar support.

\section{ACKNOWLEDGMENTS}

The author offers many thanks to Drs. Peter Mees, Christiane Reitz, and Gesine Krüger for their support for data analysis on German Soldiers' needs.

\section{AUTHOR DISCLOSURE STATEMENT}

Arndt Büssing, MD, is a professor at the Witten/Herdecke University, and was appointed to the NATO panel HFM-195 ("Integrative Medicine Interventions for Military Personnel") as a technical team member for the duration of the Team. The travel expenses to the team meetings were supported by resources from the German Ministry of Defense. The author did not receive any funding by governmental or non-governmental organizations to write this report. The author was never employed or paid by any military or religious organization. No competing financial conflicts exist.

\section{REFERENCES}

1. Jonas WB, O'Connor FG, Deuster P, Peck J, Shake C, Frost SS. Why total force fitness? Mil Med. 2010;175(8):6-13.

2. Hufford DJ, Fritts MJ, Rhodes JE. Spiritual fitness. Mil Med. 2010;175(8):73-87.

3. Büssing A. Measures of spirituality in health care. In: Cobb MR, Puchalski CM, Rumbold B, eds. Oxford Textbook of Spirituality in Healthcare. Oxford: Oxford University Press; 2012:323-331.

4. Tanyi RA. Towards clarification of the meaning of spirituality. J Adv Nurs. 2002;39(5):500-509.

5. Underwood LG, Teresi JA. The Daily Spiritual Experience Scale: Development, theoretical description, reliability, exploratory factor analysis, and preliminary construct validity using health-related data. Ann Behav Med. 2002;24(1):22-33.

6. Pargament KI. The Psychology of Religion and Coping: Theory, Research, Practice. New York: Guilford Press; 1997.

7. Büssing A. Health-related quality of life and trust in God's help. In: Michalos AC, ed. Encyclopedia of Quality of Life Research. Dordrecht: Springer Netherlands; 2014:2801-2807.

\footnotetext{
$\dagger$ The Spiritual Needs Questionnaire (SpNQ) can be used as an assessment instrument and can be obtained by the author of this report.
} 
8. Wester FE. Soldier Spirituality in a Combat Zone: Preliminary Findings About Correlations with Ethics and Resiliency. The Army's Excellence in Character, Ethics, and Leadership (EXCEL) survey. The International Society for Military Ethics (ISME). Online document at: isme.tamu.edu/ ISME11/Wester-ISME2011.pdf Accessed May 14, 2010.

9. Koenig HG, King D, Carson VB. Handbook of Religion and Health, 2nd ed. Oxford, UK: Oxford University Press; 2010.

10. Moreira-Almeida A, Neto FL, Koenig HG. Religiousness and mental health: A review. Rev Bras Psiquiatr. 2006;28(3): 242-250.

11. Sawatzky R, Ratner PA, Chiu L. A meta-analysis of the relationship between spirituality and quality of life. Social Indicators Res. 2005;72(2):153-188.

12. Cummings JP, Pargament KI. Medicine for the spirit: Religious coping in individuals with medical conditions. $R e$ ligions. 2010;1(1):28-53.

13. Thun Thuné-Boyle IC, Stygall JA, Keshtgar MR, Newman SP. Do religious/spiritual coping strategies affect illness adjustment in patients with cancer? A systematic review of the literature. Soc Sci Med. 2006;63(1):151-164.

14. Chida Y, Steptoe A, Powell LH. Religiosity/spirituality and mortality: A systematic quantitative review. Psychother Psychosom. 2009;78(2):81-90.

15. Hoff A, Johannessen-Henry CT, Ross L, Hvidt NC, Johansen C. Religion and reduced cancer risk: What is the explanation? A review. Eur J Cancer. 2008;44(17):2573-2579.

16. Wiechman Askay S, Magyar-Russell G. Post-traumatic growth and spirituality in burn recovery. Int Rev Psychiatry. 2009;21(6):570-579.

17. Weitzner E, Surca S, Wiese S, et al. Getting on with life: Positive experiences of living with a spinal cord injury. Qual Health Res. 2011;21(11):1455-1468.

18. Büssing A, Ostermann T, Koenig HG. Relevance of religion and spirituality in German patients with chronic diseases. Int J Psychiatry Med. 2007;37(1):39-57.

19. Büssing A, Michalsen A, Balzat HJ, et al. Are spirituality and religiosity resources for patients with chronic pain conditions? Pain Med. 2009;10(2):327-339.

20. Zwingmann C, Wirtz M, Müller C, Körber J, Murken S. Positive and negative religious coping in German breast cancer patients. J Behav Med. 2006;29(6):533-547.

21. Zwingmann C, Müller C, Körber J, Murken S. Religious commitment, religious coping and anxiety: A study in German patients with breast cancer. Eur J Cancer Care (Engl). 2008;17(4):361-370.

22. Büssing A, Koenig HG. The BENEFIT through spirituality/ religiosity scale-A 6-item measure for use in health outcome studies. Int J Psychiatry Med. 2008;38(4):493-506.

23. Büssing A, Föller-Mancini A, Gidley J, Heusser P. Aspects of spirituality in adolescents. Int J Child Spiritual. 2010;15(1):25-44.

24. Balboni TA, Vanderwerker LC, Block SD, et al. Religiousness and spiritual support among advanced cancer patients and associations with end-of-life treatment preferences and quality of life. J Clin Oncol. 2007;25(5):555-560.

25. Balboni TA, Paulk ME, Balboni MJ, et al. Provision of spiritual care to patients with advanced cancer: Associations with medical care and quality of life near death. J Clin Oncol. 2010;28(3):445-452.
26. Büssing A, Koenig HG. Spiritual needs of patients with chronic diseases. Religions. 2010;1(1):18-27.

27. Moadel A, Morgan C, Fatone A, et al. Seeking meaning and hope: Self-reported spiritual and existential needs among an ethnically-diverse cancer patient population. Psychooncology. 1999;8(5):378-385.

28. Büssing A, Janko A, Baumann K, Hvidt NC, Kopf A. Spiritual needs among patients with chronic pain diseases and cancer living in a secular society. Pain Med. 2013;14(9):1362-1373.

29. Büssing A, Zhai XF, Peng WB, Ling CQ. Psychosocial and spiritual needs of patients with chronic diseases: Validation of the Chinese version of the Spiritual Needs Questionnaire. $J$ Integr Med. 2013;11(2):106-115.

30. Büssing A, Pilchowska I, Surzykiewicz J. Spiritual needs of Polish patients with chronic diseases. J Relig Health. 2015;54(5):1524-1542.

31. Wansink B, Wansink CS. Are there atheists in foxholes? Combat intensity and religious behavior. J Relig Health. 2013;52(3):768-779.

32. Hourani LL, Williams J, Forman-Hoffman V, Lane ME, Weimer B, Bray RM. Influence of spirituality on depression, posttraumatic stress disorder, and suicidality in active duty military personnel. Depress Res Treat. 2012;2012:425463.

33. Büssing A, Recchia DR. Spiritual and non-spiritual needs among German soldiers and their relation to stress perception, PTSD symptoms, and life satisfaction-results from a structural equation modeling approach. J Relig Health. 2015; June 2015 (DOI 10.1007/s10943-015-0073-y).

34. Hasanović M, Pajević I. Religious moral beliefs inversely related to trauma experiences [sic] severity and depression severity among war veterans in Bosnia and Herzegovina. $J$ Relig Health. 2013;52(3):730-739.

35. Bonner LM, Lanto AB, Bolkan C, et al. Help-seeking from clergy and spiritual counselors among veterans with depression and PTSD in primary care. J Relig Health. 2013;52(3):707-718.

36. Edelson R, Soldzs S. Does Comprehensive Soldier Fitness Work? CSF Research Fails the Test. Coalition for an Ethical Psychology, Working Paper Number 1, May 2012. Online document at: www.ethicalpsychology.org Accessed November 27, 2014.

37. Büssing A, Balzat HJ, Heusser P. Spiritual needs of patients with chronic pain diseases and cancer-validation of the Spiritual Needs Questionnaire. Eur J Med Res. 2010;15(6):266-273.

38. Lin HR, Bauer-Wu SM. Psycho-spiritual well-being in patients with advanced cancer: An integrative review of the literature. J Adv Nurs. 2003;44(1):69-80.

39. Puchalski C, Ferrell B, Virani R, et al. Improving the quality of spiritual care as a dimension of palliative care: The report of the Consensus Conference. J Palliat Med. 2009;12(10):885-904.

Address correspondence to: Arndt Büssing, $M D$ Institute of Integrative Medicine Witten/Herdecke University, Gerhard-Kienle-Weg 4 58313 Herdecke Germany

E-mail: arndt.buessing@uni-wh.de 